\title{
A study to correlate the endoscopic and CT findings in chronic rhinosinusitis
}

\author{
Ashiya Goel ${ }^{1}$, Aman $^{2}$, Vikasdeep Gupta ${ }^{3, *}, V_{i n n y}$ Raheja $^{4}$, Shruti Goel $^{5}$ \\ 1,2,4,5 Junior Resident, ${ }^{3}$ Senior Resident, Dept. of ENT, ${ }^{1-4}$ Pandit Bhagwat Dayal Sharma Post Graduate Institute of Medical Sciences, \\ Rohtak, Haryana, ${ }^{5}$ Government Medical College, Haldwani, Uttarakhand, India
}

*Corresponding Author: Vikasdeep Gupta

Email: vdgupta88@gmail.com

\begin{abstract}
Nasal endoscopy and computed tomography (CT) scans are used as diagnostic modalities of paranasal sinus disease. A randomized study was carried over 40 patients diagnosed with chronic rhinosinusitis who undervent a diagnostic nasal endoscopy and a CT scan. The purpose of the study was to demonstrate the effectiveness and limitations of CT and endoscopy in assessment of CRS. In this study we observed that endoscopy was superior for assessment of localized disease like polyp, pathological secretion, presence of accessory maxillary ostia and condition of mucosa. CT scan gave a better idea of the condition of paranasal sinuses, the osteomeatal complex and various anatomical variations including concha bullosa, paradoxical middle turbinate and deviated nasal septum.
\end{abstract}

Keywords: Tomography, Endoscopy.

\section{Introduction}

Chronic rhinosinusistis (CRS) is rapidly evolving entity. There has been an exponential increase in the cases with CRS due to the increasing amount of atmospheric pollution and the exposure to numerous different allergens. CRS is one of the common reasons for ENT consultation today, hampering the patients' quality of life. ${ }^{1}$ Chronic Rhinosinusitis is defined as longstanding inflammation of nasal and paranasal mucosa as lasting more than 12 weeks and its diagnosis is based on the presence of major and minor factors. Twelve weeks or longer of two or more of the following signs and symptoms:

1. Mucopurulent nasal discharge (anterior, posterior, or both);

2. Nasal obstruction;

3. Sensation of facial pain, pressure or fullness; or

4. Decreased sense of smell.

Due to wide discrepancies in the diagnosis based on symptoms alone, objective measures gained importance in the diagnosis of CRS. The presence of middle meatal inflammation was a rationale addition to the diagnostic criteria for CRS to upgrade its diagnostic accuracy.,3

Objective measures include:

1. Purulent mucoid secretions or oedema in the middle meatus or ethmoid region;

2. Polyps in nasal cavity or in the middle meatus;

3. Inflammation of the paranasal sinuses displayed by radiographic imaging;

Various anatomical variations of the osteomeatal complex (OMC) and the paranasal sinuses have been proved to be associated with chronic and recurrent sinusitis, and radiological imaging with a Computed Tomographic (CT) scan has become an important diagnostic tool. Most patients of CRS show changes on both endoscopy and CT. However, a few may present with symptoms and endoscopic examination suggestive of a sinonasal disease, with minor abnormalities on CT scan. ${ }^{4}$ The OMC is the most important area involved in the pathogenesis of chronic rhinosinusitis. ${ }^{5}$ The knowledge of mucocilliary drainage pattern and pathophysiology of paranasal sinus disease are the keys to functional endoscopic sinus surgery (FESS). Currently, FESS is advised for patients who were unresponsive to optimum medical therapy.

According to current data, a preoperative endoscopy alone does not suffice and obviate the need for a CT scan. The diagnostic evaluation of the patient posted for FESS consists of a combination of both nasal endoscopy and CT scan. So this study was carried out to evaluate the accuracy of nasal endoscopy and to compare it with gold standard diagnostic modality namely sinus CT scan.

\section{Material and Methods}

A diligent history was taken. A history consistent with the diagnosis of chronic rhinosinusitis includes the presence of two or more major factors or one major and two minor factors or three minor factors.

\section{Major factor}

1. Facial pain or pressure

2. Nasal congestion

3. Nasal obstruction

4. Nasal discharge

5. Purulence

6. Post nasal discharge

7. Hyposmia or Anosmia

Minor factor

1. Headache

2. Fever

3. Halitosis

4. Dental pain

5. Cough

6. Ear pain or fullness

A total of 40 cases of CRS (diagnosed based on their symptoms) refractory to medical treatment in the age group of 15 to 65 years were investigated and subjected to the study during a period of one year. Patients with history of trauma, dental surgery and sinus malignancy were excluded.

In all patients first a diagnostic nasal endoscopy was performed in the office with a 0 degree and 30 degree $4 \mathrm{~mm}$ 
endoscope. Endoscopic findings suggestive of mucosal edema, watery or purulent discharge and nasal polyps either alone or in combination were considered positive. The recordings were then quantified using the Lund-Kennedy scoring system. ${ }^{6}$ A Lund Kennedy endoscopic score $\geq 2$ was diagnostic of CRS.

Each patient then underwent a plain CT scan PNS with coronal, axial and sagittal sections (1 $\mathrm{mm}$ cuts). All anatomical variations were identified and CT scan was staged according to Lund Mackay CT scoring system. ${ }^{7}$ A score $\geq 4$ was considered positive. Data for anatomical variations, endoscopic findings and CT scores was then tabulated.



Fig. 1: Concha bullosa
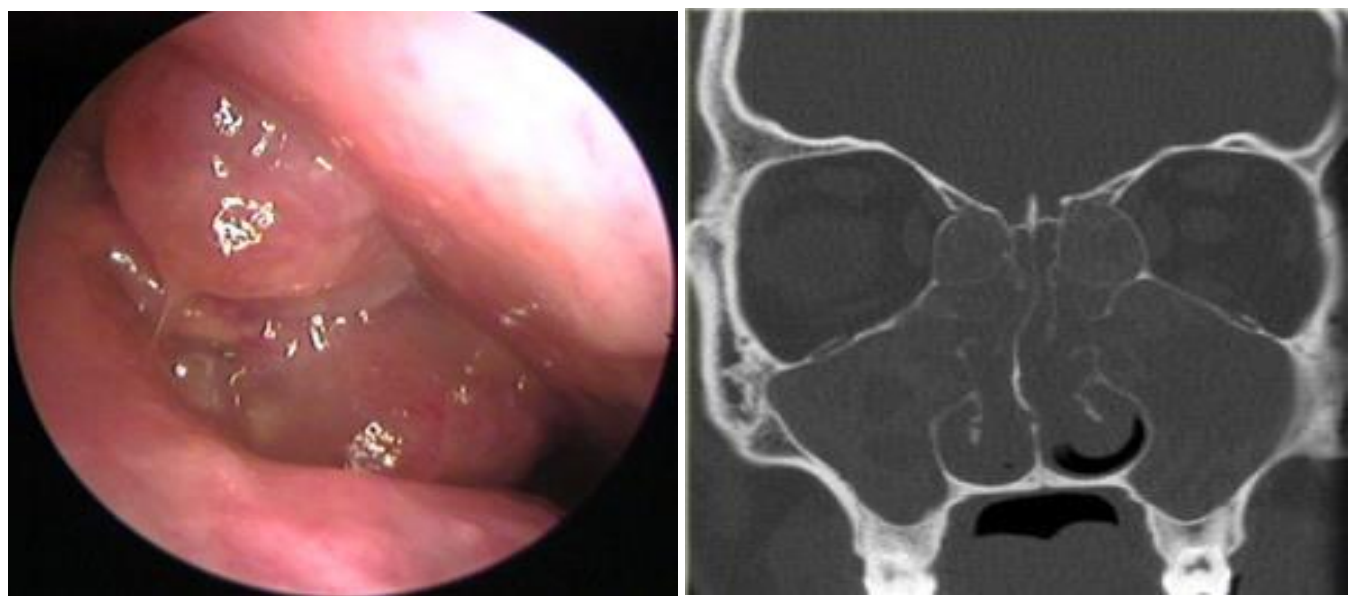

Fig. 2: Polyps

\section{Observation and Results}

Among the 40 clinically diagnosed patients of CRS, 17 were endoscopy positive for anatomic variations while 23 were CT scan positive. The correlation was statistically significant with pearson chi-square value: 24.41 and pvalue $=.0001$ i.e. $<0.05$.

Furthermore, nasal endoscopy had sensitivity: $76.47 \%$ (95\% c.i.: $66.57 \%-86.58 \%$ ) specificity: $92.86 \%$ (95\% c.i: $30.91 \%-90.95 \%)$, positive predictive value: $98.11 \%$, negative predictive value: $44.82 \%$. Thirty four patients showed mucosal changes in nasal endoscopy while only 10 in CT Scan. Osteo meatal complex blockage was also more diagnosed better with nasal endoscopy.

CT is able to identify turbinate pneumatisation more accurately as compared to nasal endoscopy.

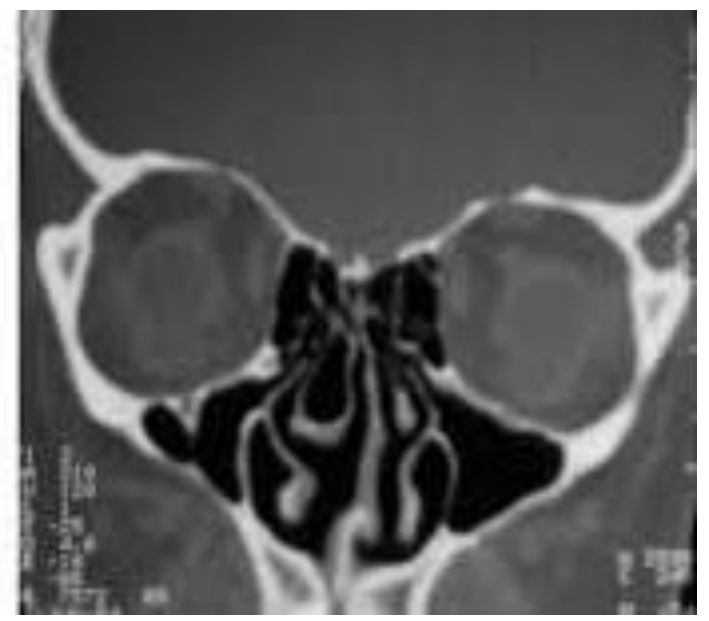




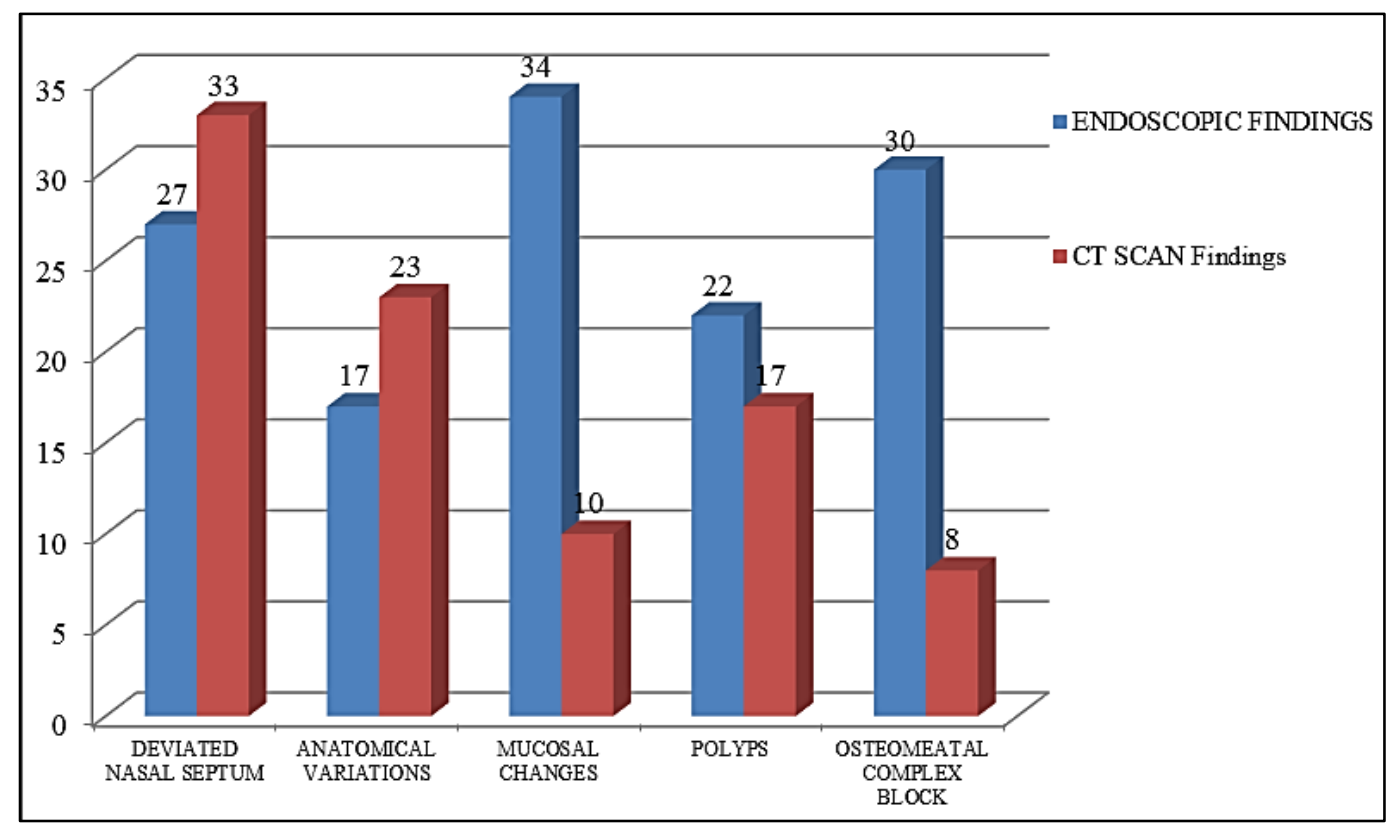

Fig. 3

\section{Discussion}

CT scan can prove to be helpful in identifying any sinonasal disease especially in inaccessible and hidden areas during nasal endoscopy. When coupled with nasal endoscopy, CT scan provides most of the objective data needed for diagnosing CRS. ${ }^{8}$ The aim of this study was to determine the accuracy of endoscopic findings in recognizing patients with CRS on comparison with CT scan. The results in our study suggested that for most of the cases, there is a high level of agreement between the results of the two modalities. Endoscopy is comparable to CT for diagnosing CRS in accessible areas especially local changes which are better appreciated during endoscopy. Similar results were obtained by few other studies. Ferguson et al in 2012 studied 125 patients of CRS concluded that nasal endoscopy has high specificity $(100 \%)$ and low sensitivity (24\%) which make it useful for confirming CRS diagnosis but not for ruling it out. ${ }^{9}$

Lohiya et al in 2014 studied 100 patients and observed that high sensitivity (88.04\%), and PPV (94.19\%) render nasal endoscopy as a good approach to accurately diagnose the disease and has strong correlation with CT. ${ }^{10}$ According to Shahizon et al. 2008 minor bony deviations can be seen on CT scan while endoscopy documents nasal septal deviation only if it is obstructive as even minor deviations i.e. less than $5 \mathrm{~mm}$ were considered significant on CT. ${ }^{11}$ Pandey et al in 2014 reached to a conclusion that accessory maxillary ostium (increased in number in CRS patients) is visualised only on endoscopy. ${ }^{12}$

\section{Conclusion}

We observed that nasal endoscopy has distinct advantages over CT for assessment of localised changes like polyps, pathological secretions, and the condition of nasal mucosa while CT gives a better idea of the condition of paranasal sinuses and the osteomeatal complex. In a few cases where it is not possible to pass the endoscope beyond a certain point due to the presence of gross pathologies like a severe deviated septum, there CT proves more helpful. CT scan depicts the extent of disease, anatomical and pathological variations far better than any other method. Endoscopy is superior to CT for identifying mucosal changes. A negative endoscopy has a strong association with CT findings and the two modalities are complimentary to each other.

\section{References}

1. Osgruthrope JD, Hadley JA; Rhinosinusitis; Current concepts in diagnosis \& management. Med Clin North AM 1999;83:2742.

2. Lanza DC, Kennedy DW. Adult rhinosinusitis defined. Otolaryngol Head Neck Surg 1997;117:S1-7.

3. Rosenfeld RM, Andes D, Bhattacharyya N, Cheung D, Eisenberg S, Ganiats TGS et al. Clinical practice guideline. Otolaryngol Head Neck Surg 2007:137:S1-31.

4. Vining EM, Yanagisawa K, Yanagisawa E. The importance of preoperative nasal endoscopy in patients with sinonasal disease. Laryngoscope 1993;103:512-519.

5. Llyod GAS, Lund VJ, Scadding GK. CT of the paranasal sinuses and functional endoscopic surgery: a critical analysis of 100 symptomatic patients. J Laryngol Otol 1991;105:181185.

6. Lund VJ, Kennedy DW. Quantification for staging sinusitis. The Staging and Therapy Group. Ann Otol Rhinol Laryngol Suppl 1995;167:17-21.

7. Hopkins Claire. The Lund-Mackay staging system for chronic rhinosinusitis: How is it used and what does it predict? Otolaryngol Head Neck Surg 2007;137:555-561.

8. Bolger W, Butzin C, Parsons D. Paranasal sinus bony anatomic variations and mucosal abnormalities: $\mathrm{CT}$ analysis for endoscopic sinus surgery. Laryngoscope 1991;101:56-64.

9. Ferguson BJ, Narita M, Yu VL, Wagener MM, Gwaltney JM Jr. Prospective observational study of chronic rhinosinusitis: environmental triggers and antibiotic implications. Clin Infect Dis 2012;54:62-68. 
10. Lohiya SS, Patel SV, Pawde AM, Bokare BD, Sakhare PT. Comparative Study of Diagnostic Nasal Endoscopy and CT Paranasal Sinuses in Diagnosing Chronic Rhinosinusitis. Indian J Otolaryngol Head Neck Surg 2016; 68:224-229.

11. Shahizon AMM, Suraya A, Rozman Z, Aini AA, Gendeh BS Correlation of Computed Tomography and Nasal Endoscopic Findings in Chronic Rhino sinusitis. Med J Malaysia 2008;63:211-215.

12. Pandey A, Nigam R, Jain AK. A Comparative Study of Radiological and Endoscopic Findings in Paranasal Sinus Disease. Sch J App Med Sci 2014;2:1053-1058.

How to cite this article: Goel A, Aman, Gupta V, Raheja V, Goel S. A study to correlate the endoscopic and CT findings in chronic rhinosinusitis. Indian $J$ Anat Surg Head Neck Brain 2018;4(4):114-117. 